\title{
Dissipativity-Based Controller Design for Time-Delayed T-S Fuzzy Switched Distributed Parameter Systems
}

\author{
Xiaona Song $\mathbb{D}^{\mathrm{D}},{ }^{1}$ Mi Wang $\left(\mathbb{D},{ }^{1}\right.$ Shuai Song, ${ }^{2}$ and Jingtao $\mathrm{Man}^{1}$ \\ ${ }^{1}$ School of Information Engineering, Henan University of Science and Technology, Luoyang 471023, China \\ ${ }^{2}$ School of Automation, Nanjing University of Science and Technology, Nanjing 210094, China \\ Correspondence should be addressed to Xiaona Song; xiaona_97@163.com
}

Received 1 May 2018; Accepted 30 September 2018; Published 17 October 2018

Academic Editor: Michele Scarpiniti

Copyright (c) 2018 Xiaona Song et al. This is an open access article distributed under the Creative Commons Attribution License, which permits unrestricted use, distribution, and reproduction in any medium, provided the original work is properly cited.

\begin{abstract}
This paper studies fuzzy controller design problem for a class of nonlinear switched distributed parameter systems (DPSs) subject to time-varying delay. Initially, the original nonlinear DPSs are accurately described by Takagi-Sugeno fuzzy model in a local region. On the basis of parallel distributed compensation technique, mode-dependent fuzzy proportional and fuzzy proportional-spatialderivative controllers are constructed, respectively. Subsequently, using single Lyapunov-Krasovskii functional and some matrix inequality methods, sufficient conditions that guarantee the stability and dissipativity of the closed-loop systems are presented in the form of linear matrix inequalities, which allow the control gain matrices to be easily obtained. Finally, numerical examples are provided to demonstrate the validity of the designed controllers.
\end{abstract}

\section{Introduction}

Different from lumped parameter systems, the dynamic behaviors of many industrial processes depend on not only time but also the space, for which the mathematical models are described by various partial differential equations (PDEs) such as parabolic, hyperbolic, elliptic, etc. [1]. In the past decades, considerable research has been conducted for distributed parameter systems (DPSs), especially hyperbolic and parabolic PDE systems [2-10]. To mention a few, the authors in [2] investigated the state estimation problem of linear parabolic PDE systems via using backstepping technique, [3] considered the robust controller design for hyperbolic DPSs, adaptive control approach of parabolic PDE systems was proposed in [4-6], and [10] investigated boundary control problem of parabolic PDE systems with parameter variations. However, most of the mentioned works are reported for linear PDE systems, and the control of nonlinear DPSs is still an open issue.

Since Takagi-Sugeno (T-S) fuzzy system was proposed, a huge body studies for nonlinear ordinary differential equation (ODE) systems have been carried out [11-20]. Inspired by the processing method of nonlinear ODE systems, based on T-S fuzzy model, a few results have been obtained for nonlinear PDE systems recently. For instances, Wu et al. [2123] realized exponential stabilization for a class of nonlinear parabolic PDE systems via designing a fuzzy controller and illustrated the application on nonisothermal plug-flow reactor, Zhu et al. [24] developed fuzzy control method subject to a class of nonlinear coupled ODE-PDE systems with input constraint.

It should be pointed out that switched PDE systems have not been paid enough attention. Switched system (SS) is an important class of hybrid systems and plays a key role in industrial process. SSs consist of numbers of subsystems, in which switching rule is utilized to indicate the active subsystem at each instant of time. For switched systems, switching signals are classified into arbitrary switching signal, timedependent signal, and state-dependent switching signal. As the fundamental problem, the stabilization of switched PDEs has been investigated in $[25,26]$ and semiconductor power chip is used to illustrate the practical application, whereas, to the best of our knowledge, the control synthesis problem for switched PDE systems has not been fully investigated, which motivates us to do this work.

On another research front, proportional-spatialderivative $(\mathrm{P}-\mathrm{sD})$ controller has been considered in many DPSs [27-29] because of its advantages compared with 
proportional $(\mathrm{P})$ controller. Thus, in this paper, we investigate the problem of control synthesis for switched nonlinear parabolic PDE systems with time delay, including both fuzzy $\mathrm{P}$ and $\mathrm{P}-\mathrm{sD}$ control approaches. Based on single LyapunovKrasovskii functional and Wirtinger's inequality, sufficient conditions for stabilization and dissipative performance are obtained by using linear matrix inequalities. Finally, simulation studies are presented to show the effectiveness of the proposed strategies.

We organize the framework as follows: The model description is given in Section 2, controllers designs are presented in Section 3, and Section 4 provides simulations to illustrate the validity of the proposed control design approaches. Finally, conclusions are offered in Section 5.

Notations. The notation that have been used in this paper are fairly standard. $\mathbb{R}$ and $\mathbb{R}^{n}$ denote the set of all real numbers and $n$ dimensional Euclidean space. $*$ stands for symmetric completion, for example, $A+*=A+A^{\mathrm{T}}$. $\|\omega(x)\|_{2}=\sqrt{\int_{l_{1}}^{l_{2}} \omega^{\mathrm{T}}(x) \omega(x) d x} \cdot \mathrm{H}_{n}^{l}\left(l_{1}, l_{2}\right)=W^{l, 2}\left(\left(l_{1}, l_{2}\right) ; \mathbb{R}^{n}\right)$ is a real Sobolve space of absolutely continuous vector functions $\omega(x):\left(l_{1}, l_{2}\right) \longrightarrow \mathbb{R}^{n}$ with square integrable derivatives $d^{l} \omega(x) / d x^{l}$ of the order $l \geq 1$ and

$$
\|\omega(x)\|_{\mathrm{H}_{n}^{l}\left(l_{1}, l_{2}\right)}=\sqrt{\int_{l_{1}}^{l_{2}} \sum_{i=0}^{l}\left(\frac{d^{i} \omega(x)}{d x^{i}}\right)^{\mathrm{T}}\left(\frac{d^{i} \omega(x)}{d x^{i}}\right) d x .}
$$

\section{Preliminary System and T-S Fuzzy Model}

In this note, a class of switched nonlinear distributed parameter systems with time delay is investigated, which can be described by the following parabolic partial differential equations (PDEs):

$$
\begin{aligned}
y_{t}(x, t)= & \Theta y_{x x}(x, t)+f_{\sigma 1}(y(x, t)) \\
& +f_{\sigma d}(y(x, t-\tau(t))) \\
& +g_{\sigma u}(y(x, t)) u(x, t) \\
& +g_{\sigma \omega}(y(x, t)) \omega(x, t) \\
z(x, t)= & f_{\sigma 2}(y(x, t))
\end{aligned}
$$

subject to the Dirichlet boundary condition

$$
y\left(l_{1}, t\right)=y\left(l_{2}, t\right)=0
$$

and the initial condition

$$
y(x, t)=y_{0}(x)
$$

where $y(x, t) \in \mathbb{R}^{n}$ denotes the state vector; $x \in\left[l_{1}, l_{2}\right] \subset \mathbb{R}$ and $t \in[0, \infty) \subset \mathbb{R}$ are the spatial position and time, respectively. $z(x, t) \in \mathbb{R}^{n}$ represents the output, $u(x, t) \in \mathbb{R}^{n}$ is the control input, $\omega(x, t) \in \mathbb{R}^{n}$ is the disturbance satisfying $\int_{0}^{\infty}\|\omega(x, t)\|_{2}^{2} d t<\infty$, and $\tau(t)$ is the time delay, which satisfies $0 \leq \tau(t) \leq h, 0 \leq \dot{\tau}(t) \leq d \leq 1$, and $\Theta$ is a known matrix. For convenience, define

$$
\mathscr{A} y(x, t)=\Theta y_{x x}(x, t)
$$

Based on sector nonlinearity method, the switched nonlinear parabolic PDE systems (2) can be represented by Takagi-Sugeno fuzzy switched dynamic model as follows.

Plant Rule $\Re_{\sigma}^{i}$ : THEN

IF: $\theta_{1}(x, t)$ is $F_{\sigma 1}^{i}, \theta_{2}(x, t)$ is $F_{\sigma 2}^{i}, \ldots$, and $\theta_{p}(x, t)$ is $F_{\sigma p}^{i}$,

$$
\begin{aligned}
y_{t}(x, t)= & \mathscr{A} y(x, t)+A_{\sigma i} y(x, t)+A_{d \sigma i} y(x, t-\tau(t)) \\
& +B_{\sigma i} u(x, t)+C_{\sigma i} \omega(x, t) \\
z(x, t)= & L_{\sigma i} y(x, t)
\end{aligned}
$$

where $\theta_{j}(x, t)$ are premise variables and $F_{\sigma j}^{i}$ are fuzzy sets $(i \in\{1,2, \ldots, s\}, j \in\{1,2, \ldots, p\}) . \sigma \in F=\{1,2, \ldots, l\}$ is a switching signal, which is a piecewise constant function.

For fuzzy controller design, we represent the fuzzy switched systems as follows:

$$
\begin{aligned}
& y_{t}(x, t)=\mathscr{A} y(x, t)+\sum_{i=1}^{s} h_{\sigma i}(\theta(x, t)) \times\left[A_{\sigma i} y(x, t)\right. \\
& \left.+A_{d \sigma i} y(x, t-\tau(t))+B_{\sigma i} u(x, t)+C_{\sigma i} \omega(x, t)\right] \\
& z(x, t)=\sum_{i=1}^{s} h_{\sigma i}(\theta(x, t)) L_{\sigma i} y(x, t)
\end{aligned}
$$

where $h_{\sigma i}(\theta(x, t))=\prod_{j=1}^{p} F_{\sigma j}^{i}\left(\theta_{j}(x, t)\right) / \sum_{i=1}^{s} \prod_{j=1}^{p} F_{\sigma j}^{i}\left(\theta_{j}(x, t)\right)$, $0<h_{\sigma i}(\theta(x, t))<1$, and $\sum_{i=1}^{s} h_{\sigma i}(\theta(x, t))=1$ denote the membership functions with $\theta_{j}(x, t)$ belonging to the fuzzy set $F_{\sigma j}^{i}$.

In this study, it is assumed that the switching signal $\sigma$ is arbitrary subjects to $\nu_{r}(t)=1,0(r \in F)$, which means that the $r$-th subsystem is active or not, respectively. Thus the fuzzy switched PDE system (7) can be inferred as follows:

$$
\begin{aligned}
& y_{t}(x, t)=\mathscr{A} y(x, t)+\sum_{r=1}^{l} \sum_{i=1}^{s} v_{r}(t) h_{r i}(\theta(x, t)) \\
& \quad \times\left[A_{r i} y(x, t)+A_{d r i} y(x, t-\tau(t))+B_{r i} u(x, t)\right. \\
& \left.\quad+C_{r i} \omega(x, t)\right] \\
& z(x, t)=\sum_{r=1}^{l} \sum_{i=1}^{s} v_{r}(t) h_{r i}(\theta(x, t)) L_{r i} y(x, t)
\end{aligned}
$$

The following definition and lemma will be used to obtain the main results.

Definition 1. Given matrices $R_{1}=R_{1}^{\mathrm{T}}, R_{2}=R_{2}^{\mathrm{T}}$, system (8) is said to be dissipative if the following inequality holds for a nonnegative real function $V(\cdot)$ with $V(0)=0$, called the storage function,

$$
\begin{aligned}
& \int_{0}^{\tau} \int_{l_{1}}^{l_{1}}\left[z^{\mathrm{T}}(x, t) R_{1} z(x, t)+2 z^{\mathrm{T}}(x, t) S \omega(x, t)\right. \\
& \left.\quad+\omega^{\mathrm{T}}(x, t) R_{2} \omega(x, t)\right] d x d t+V\left(y_{0}(x)\right) \\
& \quad \geq 0, \quad \forall \tau \geq 0
\end{aligned}
$$

where $y_{0}(x)=y(x, 0)$. 
Lemma 2 ([30], vector-valued Wirtinger's inequalities). Let $y \in \mathrm{H}_{n}^{l}\left(l_{1}, l_{2}\right)$ be a vector function with $y\left(l_{1}\right)=y\left(l_{2}\right)=0$, then for any $n \times n$ real matrix $E \geq 0$, we have

$$
\begin{aligned}
& \int_{l_{1}}^{l_{2}} y(s) E y(s) d s \\
& \quad \leq\left(l_{2}-l_{1}\right)^{2} \pi^{-2} \int_{l_{1}}^{l_{2}}\left(\frac{d y(s)}{d s}\right)^{\mathrm{T}} E\left(\frac{d y(s)}{d s}\right) d s
\end{aligned}
$$

Moreover, if $y\left(l_{1}\right)=0$ or $y\left(l_{2}\right)=0$, then

$$
\begin{aligned}
& \int_{l_{1}}^{l_{2}} y(s) E y(s) d s \\
& \quad \leq 4\left(l_{2}-l_{1}\right)^{2} \pi^{-2} \int_{l_{1}}^{l_{2}}\left(\frac{d y(s)}{d s}\right)^{\mathrm{T}} E\left(\frac{d y(s)}{d s}\right) d s
\end{aligned}
$$

\section{Fuzzy Controllers Design}

In this section, two different fuzzy controllers will be proposed for the fuzzy switched distributed parameter system (8).

Firstly, we consider designing a proportional control law in Section 3.1.

3.1. Fuzzy Proportional Controller Design. Using parallel distributed compensation (PDC) strategy, the following fuzzy controller is considered:

$$
u(x, t)=\sum_{r=1}^{l} \sum_{i=1}^{s} v_{r}(t) h_{r i}(\theta(x, t)) K_{r i} y(x, t)
$$

Then, from (8) and (12), the following system is got:

$$
\begin{aligned}
& y_{t}(x, t)=\mathscr{A} y(x, t) \\
& \quad+\sum_{r=1}^{l} \sum_{i=1}^{s} \sum_{j=1}^{s} v_{r}(t) h_{r i}(\theta(x, t)) h_{r j}(\theta(x, t)) \\
& \times\left[A_{r i} y(x, t)+A_{d r i} y(x, t-\tau(t))+B_{r j} K_{r i} y(x, t)\right. \\
& \left.\quad+C_{r i} \omega(x, t)\right] \\
& z(x, t)=\sum_{r=1}^{l} \sum_{i=1}^{s} v_{r}(t) h_{r i}(\theta(x, t)) L_{r i} y(x, t)
\end{aligned}
$$

Theorem 3. For the fuzzy switched system (13), if there exist symmetric positive matrices $P, Q$ and scalars $\varepsilon_{r i}(i, j=$ $1,2, \ldots, s, r=1,2, \ldots, l)$ such that

$$
P \Theta+*>0
$$

and

$$
\left(\begin{array}{ccc}
\Xi_{r i j} & P A_{d r i} & P C_{r i}-L_{r i}^{\mathrm{T}} S \\
* & -(1-d) Q & 0 \\
* & * & -R_{2}
\end{array}\right)<0
$$

where

$$
\begin{aligned}
\Xi_{r i j}= & {\left[P A_{r i}+\varepsilon_{r i} P B_{r j} B_{r i}^{\mathrm{T}} P-\pi^{2}\left(l_{2}-l_{1}\right)^{-2} P \Theta\right]+* } \\
& -L_{r i}^{\mathrm{T}} R_{1} L_{r i}+Q \\
K_{r i}= & \varepsilon_{r i} B_{r i}^{\mathrm{T}} P
\end{aligned}
$$

then for any switching signals, the controller (12) can guarantee that the fuzzy switched system (13) is asymptotically stable with dissipativity performance subject to given matrices $S, R_{1}<0$, and $R_{2}=R_{2}^{\mathrm{T}}$.

Proof. According to Green's formula, Lemma 2, and Dirichlet boundary condition, we have

$$
\begin{aligned}
& 2 \int_{l_{1}}^{l_{2}} y^{\mathrm{T}}(x, t) P \mathscr{A} y(x, t) d x \\
& \quad=-\int_{l_{1}}^{l_{2}} y_{x}^{\mathrm{T}}(x, t)[P \Theta+*] y_{x}(x, t) d x \\
& \quad \leq-\pi^{2}\left(l_{2}-l_{1}\right)^{-2} \int_{l_{1}}^{l_{2}} y^{\mathrm{T}}(x, t)[P \Theta+*] y(x, t) d x
\end{aligned}
$$

For convenience, define

$$
\begin{gathered}
A_{h r i}=\sum_{r=1}^{l} \sum_{i=1}^{s} v_{r}(t) h_{r i}(\theta(x, t)) A_{r i} \\
C_{h r i}=\sum_{r=1}^{l} \sum_{i=1}^{s} v_{r}(t) h_{r i}(\theta(x, t)) C_{r i} \\
A_{d h r i}=\sum_{r=1}^{l} \sum_{i=1}^{s} v_{r}(t) h_{r i}(\theta(x, t)) A_{d h r i}
\end{gathered}
$$

and

$$
\begin{aligned}
B_{h r j} K_{h r i} & \\
= & \sum_{r=1}^{l} \sum_{i=1}^{s} \sum_{j=1}^{s} v_{r}(t) h_{r i}(\theta(x, t)) h_{r j}(\theta(x, t)) B_{r j} K_{r i} \\
h_{r i j} & =\sum_{r=1}^{l} \sum_{i=1}^{s} \sum_{j=1}^{s} v_{r}(t) h_{r i}(\theta(x, t)) h_{r j}(\theta(x, t))
\end{aligned}
$$

Choose a Lyapunov functional with the following form:

$$
\begin{aligned}
V(t)= & \int_{l_{1}}^{l_{2}} y^{\mathrm{T}}(x, t) P y(x, t) d x \\
& +\int_{l_{1}}^{l_{2}} \int_{t-\tau(t)}^{t} y^{\mathrm{T}}(x, \alpha) Q y(x, \alpha) d \alpha d x
\end{aligned}
$$


Differentiating $V(t)$ along the solution of the system (13), we get

$$
\begin{aligned}
& \dot{V}(t) \leq 2 \int_{l_{1}}^{l_{2}} y^{\mathrm{T}}(x, t) P y_{t}(x, t) d x+\int_{l_{1}}^{l_{2}} y^{\mathrm{T}}(x, t) \\
& \cdot Q y(x, t) d x-(1-d) \int_{l_{1}}^{l_{2}} y^{\mathrm{T}}(x, t-\tau(t)) \\
& \cdot Q y(x, t-\tau(t)) d x \leq \int_{l_{1}}^{l_{2}} y^{\mathrm{T}}(x, t) \\
& \cdot\left[-\pi^{2}\left(l_{2}-l_{1}\right)^{-2} P \Theta+P A_{h r i}+P B_{h r j} K_{h r i}+*\right] \\
& \cdot y(x, t) d x+\int_{l_{1}}^{l_{2}} y^{\mathrm{T}}(x, t)\left[P A_{d h r i}+*\right] \\
& \cdot y(x, t-\tau(t)) d x+\int_{l_{1}}^{l_{2}} y^{\mathrm{T}}(x, t)\left[P C_{h r i}+*\right] \\
& \cdot \omega(x, t) d x+\int_{l_{1}}^{l_{2}} y^{\mathrm{T}}(x, t) Q y(x, t) d x-(1 \\
& -d) \int_{l_{1}}^{l_{2}} y^{\mathrm{T}}(x, t-\tau(t)) Q y(x, t-\tau(t)) d x
\end{aligned}
$$

Substituting $K_{r i}=\varepsilon_{r i} B_{r i}^{\mathrm{T}} P$ into (21), one has

$$
\begin{aligned}
& \dot{V}(t) \leq \int_{l_{1}}^{l_{2}} y^{\mathrm{T}}(x, t) \\
& \cdot\left[-\pi^{2}\left(l_{2}-l_{1}\right)^{-2} P \Theta+P A_{h r i}+\varepsilon_{r i} P B_{h r j} B_{h r i}^{\mathrm{T}} P+*\right] \\
& \cdot y(x, t) d x+\int_{l_{1}}^{l_{2}} y^{\mathrm{T}}(x, t)\left[P A_{d h r i}+*\right] \\
& \cdot y(x, t-\tau(t)) d x+\int_{l_{1}}^{l_{2}} y^{\mathrm{T}}(x, t)\left[P C_{h r i}+*\right] \\
& \cdot \omega(x, t) d x+\int_{l_{1}}^{l_{2}} y^{\mathrm{T}}(x, t) Q y(x, t) d x-(1 \\
& -d) \int_{l_{1}}^{l_{2}} y^{\mathrm{T}}(x, t-\tau(t)) Q y(x, t-\tau(t)) d x
\end{aligned}
$$

Inequality (22) can be also rewritten as follows:

$$
\begin{aligned}
\dot{V}(t) & \leq \int_{l_{1}}^{l_{2}} h_{r i j}\left(\begin{array}{c}
y(x, t) \\
y(x, t-\tau(t)) \\
\omega(x, t)
\end{array}\right) \\
& \times\left(\begin{array}{ccc}
\Psi_{r i j} & P A_{d r i} & P C_{r i} \\
* & -(1-d) Q & 0 \\
* & * & 0
\end{array}\right)\left(\begin{array}{c}
y(x, t) \\
y(x, t-\tau(t)) \\
\omega(x, t)
\end{array}\right) d x
\end{aligned}
$$

where

$$
\begin{aligned}
\Psi_{r i j}= & {\left[-\pi^{2}\left(l_{2}-l_{1}\right)^{-2} P \Theta+P A_{r i}+\varepsilon_{r i} P B_{r j} B_{r i}^{\mathrm{T}} P\right]+* } \\
& +Q
\end{aligned}
$$

To obtain the stability analysis results, we set $\omega(x, t) \equiv 0$, then the following inequality is got:

$$
\begin{aligned}
\dot{V}( & (t) \leq 2 \int_{l_{1}}^{l_{2}} y^{\mathrm{T}}(x, t) P \mathscr{A} y_{t}(x, t) d x \\
& +\int_{l_{1}}^{l_{2}} y^{\mathrm{T}}(x, t) Q y(x, t) d x-(1-d) \\
& \cdot \int_{l_{1}}^{l_{2}} y^{\mathrm{T}}(x, t-\tau(t)) Q y(x, t-\tau(t)) d x \\
& \leq-\pi^{2}\left(l_{2}-l_{1}\right)^{-2} \int_{l_{1}}^{l_{2}} y^{\mathrm{T}}(x, t)[P \Theta+*] y(x, t) d x \\
& +\int_{l_{1}}^{l_{2}} y^{\mathrm{T}}(x, t)\left[P A_{h r i}+*\right] y(x, t) d x \\
& +\int_{l_{1}}^{l_{2}} y^{\mathrm{T}}(x, t)\left[\varepsilon_{r i} P B_{h r j} B_{h r i}^{\mathrm{T}} P+*\right] y(x, t) d x \\
& +\int_{l_{1}}^{l_{2}} y^{\mathrm{T}}(x, t-\tau(t)) Q y(x, t-\tau(t)) d x \\
& +\int_{l_{1}}^{l_{2}} y^{\mathrm{T}}(x, t)\left[P A_{d h r i}+*\right] y(x, t-\tau(t)) d x \\
& +\int_{l_{1}}^{l_{2}} y^{\mathrm{T}}(x, t) Q y(x, t) d x-(1-d)
\end{aligned}
$$

Similar to the treatment in (22), inequality (25) implies that

$$
\begin{aligned}
\dot{V}(t) & \leq \int_{l_{1}}^{l_{2}} h_{r i j}\left(\begin{array}{c}
y(x, t) \\
y(x, t-\tau(t))
\end{array}\right)^{\mathrm{T}} \\
& \times\left(\begin{array}{cc}
\Psi_{r i j} & P A_{d r i} \\
* & -(1-d)
\end{array}\right)\left(\begin{array}{c}
y(x, t) \\
y(x, t-\tau(t))
\end{array}\right) d x
\end{aligned}
$$

Thus, according to Lyapunov stability theory, inequalities (14) and (15) guarantee that the fuzzy switched system (13) is stable for any switching signal. 
On the other hand, invoking (15) and (23), the following inequality is obtained:

$$
\begin{aligned}
\dot{V}(t) & -\int_{l_{1}}^{l_{2}}\left\{y^{\mathrm{T}}(x, t) L_{r i}^{\mathrm{T}} R_{1} L_{r i} y(x, t)\right. \\
& +2 y^{\mathrm{T}}(x, t) L_{r i}^{\mathrm{T}} S \omega(x, t) \\
& \left.+\omega^{\mathrm{T}}(x, t) R_{2} \omega(x, t)\right\} d x<0
\end{aligned}
$$

which can be rewritten as

$$
\begin{aligned}
\dot{V}(t) & -\int_{l_{1}}^{l_{2}}\left\{z^{\mathrm{T}}(x, t) R_{1} z(x, t)+2 z^{\mathrm{T}}(x, t) S \omega(x, t)\right. \\
& \left.+\omega^{\mathrm{T}}(x, t) R_{2} \omega(x, t)\right\} d x<0
\end{aligned}
$$

Under zero initial condition, integrating (28) on period $[0, \tau]$, then it can be derived to

$$
\begin{aligned}
0 \leq & V(\tau) \leq \int_{0}^{\tau} \int_{l_{1}}^{l_{2}}\left\{z^{\mathrm{T}}(x, t) R_{1} z(x, t)\right. \\
& +2 z^{\mathrm{T}}(x, t) S \omega(x, t) \\
& \left.+\omega^{\mathrm{T}}(x, t) R_{2} \omega(x, t)\right\} d x d t, \quad \forall \tau \geq 0
\end{aligned}
$$

It is obvious that system (13) is also dissipative. This completes the proof.

Note that the matrix inequalities in Theorem 3 is not linear; we give the following approach to solve it. Using Schur complements, we have

$$
\left(\begin{array}{cccc}
\Gamma_{r i j} & P A_{d r i} & P C_{r i}-L_{r i}^{\mathrm{T}} S & L_{r i}^{\mathrm{T}} \\
* & -(1-d) Q & 0 & 0 \\
* & * & -R_{2} & 0 \\
* & * & * & R_{1}^{-1}
\end{array}\right)<0
$$

where

$$
\begin{aligned}
\Gamma_{r i j}= & {\left[P A_{r i}+\varepsilon_{r i} P B_{r j} B_{r i}^{\mathrm{T}} P-\pi^{2}\left(l_{2}-l_{1}\right)^{-2} P \Theta\right]+* } \\
& +Q
\end{aligned}
$$

Pre- and postmultiply both sides of inequality (30) by the matrix $\operatorname{diag}\{X, X, I, I\}$, where $X=P^{-1}$. Define $\widetilde{Q}=X Q X$; then we can obtain

$$
\left(\begin{array}{cccc}
\Pi_{r i j} & A_{d r i} X & C_{r i}-X L_{r i}^{\mathrm{T}} S & X L_{r i}^{\mathrm{T}} \\
* & -(1-d) \widetilde{Q} & 0 & 0 \\
* & * & -R_{2} & 0 \\
* & * & * & R_{1}^{-1}
\end{array}\right)<0
$$

where

$$
\begin{aligned}
\Pi_{r i j}= & {\left[A_{r i} X+\varepsilon_{r i} B_{r j} B_{r i}^{\mathrm{T}}-\pi^{2}\left(l_{2}-l_{1}\right)^{-2} \Theta X\right]+* } \\
& +\widetilde{Q}
\end{aligned}
$$

The matrix $X$ and $\varepsilon_{r i}$ can be obtained by solving inequality (32); substituting $X$ and $\varepsilon_{r i}$ into

$$
K_{r i}=\varepsilon_{r i} B_{r i}^{\mathrm{T}} X^{-1}
$$

we can easily obtain the controller gains.

Remark 4. It should be emphasized that the controller designed in Theorem 3 requires distributed sensors and actuators. With the development of advanced technique, it is possible to solve this problem, because there are more and more microsensors and microactuators manufactured in control circuitry. However, we are also looking for new method to avoid the drawback of the controller.

Remark 5. It is worth pointed out that the state feedback control method (12) cannot meet the engineering needs in many practical applications, especially for nonlinear spatially distributed processes. Therefore, we will consider the following fuzzy proportional-spatial-derivative $(\mathrm{P}-\mathrm{sD})$ controller.

3.2. Fuzzy P-sD Controller Design. In contrast to the proportional state feedback controller proposed in Section 3.1, the information of state spatial-derivative is added to fuzzy $\mathrm{P}-\mathrm{sD}$ controller design, which will bring better dynamic performances.

Firstly, using PDC theory, the following fuzzy P-sD controller is constructed

$$
\begin{aligned}
& \mathcal{u}(x, t) \\
& =\sum_{r=1}^{l} \sum_{i=1}^{s} v_{r}(t) h_{r i}(\theta(x, t))\left[K_{r i} y(x, t)+K_{D r i} y_{x}(x, t)\right]
\end{aligned}
$$

Combining (8) and (35), we have

$$
\begin{aligned}
& y_{t}(x, t)=\mathscr{A} y(x, t)+\sum_{r=1}^{l} \sum_{i=1}^{s} \sum_{j=1}^{s} \nu_{r}(t) h_{r i}(\theta(x, t)) h_{r j}(\theta(x, t)) \\
& \times\left[A_{r i} y(x, t)+A_{d r i} y(x, t-\tau(t))+B_{r j} K_{r i} y(x, t)+B_{r j} K_{D r i} y_{x}(x, t)+C_{r i} \omega(x, t)\right] \\
& z(x, t)=\sum_{r=1}^{l} \sum_{i=1}^{s} v_{r}(t) h_{r i}(\theta(x, t)) L_{r i} y(x, t)
\end{aligned}
$$


Theorem 6. Given matrices $S, R_{1}<0$, and $R_{2}=R_{2}^{\mathrm{T}}$, then for arbitrary switching signals, the fuzzy switched system (36) is asymptotically stable and dissipative via using controller (35), if there exist matrices $P>0, Q>0, K_{r i}$, and $K_{\text {Dri }}$ such that the following inequality holds:

$$
\left(\begin{array}{ccccc}
\Lambda_{r i j} & P B_{r j} K_{D r i} & P A_{d r i} & P C_{r i}-L_{r i}^{\mathrm{T}} S & L_{r i}^{\mathrm{T}} \\
* & -(P \Theta+*) & 0 & 0 & 0 \\
* & * & -(1-d) Q & 0 & 0 \\
* & * & * & -R_{2} & 0 \\
* & * & * & * & R_{1}^{-1}
\end{array}\right)
$$

$$
<0
$$

where

$$
\Lambda_{r i j}=\left(P A_{r i}+P B_{r j} K_{r i}\right)+*+Q
$$

Proof. Construct the following Lyapunov functional candidate:

$$
\begin{aligned}
V(t)= & \int_{l_{1}}^{l_{2}} y^{\mathrm{T}}(x, t) P y(x, t) d x \\
& +\int_{l_{1}}^{l_{2}} \int_{t-\tau(t)}^{t} y^{\mathrm{T}}(x, \alpha) Q y(x, \alpha) d \alpha d x
\end{aligned}
$$

By differentiating $V(t)$ along the solution of system (36), we can get

$\dot{V}(t)$

$$
\begin{aligned}
\leq & 2 \int_{l_{1}}^{l_{2}} y^{\mathrm{T}}(x, t) P y_{t}(x, t) d x \\
& +\int_{l_{1}}^{l_{2}} y^{\mathrm{T}}(x, t) Q y(x, t) d x \\
& -(1-d) \int_{l_{1}}^{l_{2}} y^{\mathrm{T}}(x, t-\tau(t)) Q y(x, t-\tau(t)) d x
\end{aligned}
$$

Substituting (36) into inequality (40), one has

$$
\begin{aligned}
& \dot{V}(t) \\
& \leq 2 \int_{l_{1}}^{l_{2}} y^{\mathrm{T}}(x, t) P A y(x, t) d x \\
& \quad+\int_{l_{1}}^{l_{2}} y^{\mathrm{T}}(x, t)\left[P A_{h r i}+P B_{h r j} K_{h r i}+*\right] y(x, t) d x \\
& \quad+\int_{l_{1}}^{l_{2}} y^{\mathrm{T}}(x, t)\left[P B_{h r j} K_{\text {Dhri }}+*\right] y_{x}(x, t) d x
\end{aligned}
$$

$$
\begin{aligned}
& +\int_{l_{1}}^{l_{2}} y^{\mathrm{T}}(x, t)\left[P A_{d h r i}+*\right] y(x, t-\tau(t)) d x \\
& +\int_{l_{1}}^{l_{2}} y^{\mathrm{T}}(x, t)\left[P C_{h r i}+*\right] \omega(x, t) d x \\
& +\int_{l_{1}}^{l_{2}} y^{\mathrm{T}}(x, t) Q y(x, t) d x \\
& -(1-d) \int_{l_{1}}^{l_{2}} y^{\mathrm{T}}(x, t-\tau(t)) Q y(x, t-\tau(t)) d x
\end{aligned}
$$

According to Green's formula and Dirichlet boundary condition, it follows that

$$
\begin{aligned}
& 2 \int_{l_{1}}^{l_{2}} y^{\mathrm{T}}(x, t) P A y(x, t) d x \\
& \quad=-\int_{l_{1}}^{l_{2}} y_{x}^{\mathrm{T}}(x, t)[P \Theta+*] y_{x}(x, t) d x
\end{aligned}
$$

In light of (41) and (42), one can obtain

$$
\begin{aligned}
& \dot{V}(t) \leq-\int_{l_{1}}^{l_{2}} y_{x}^{\mathrm{T}}(x, t)[P \Theta+*] y_{x}(x, t) d x \\
& +\int_{l_{1}}^{l_{2}} y^{\mathrm{T}}(x, t)\left[P A_{h r i}+P B_{h r j} K_{h r i}+*\right] y(x, t) d x \\
& +\int_{l_{1}}^{l_{2}} y^{\mathrm{T}}(x, t)\left[P B_{h r j} K_{D h r i}+*\right] y_{x}(x, t) d x \\
& +\int_{l_{1}}^{l_{2}} y^{\mathrm{T}}(x, t)\left[P A_{d h r i}+*\right] y(x, t-\tau(t)) d x \\
& +\int_{l_{1}}^{l_{2}} y^{\mathrm{T}}(x, t)\left[P C_{h r i}+*\right] \omega(x, t) d x \\
& +\int_{l_{1}}^{l_{2}} y^{\mathrm{T}}(x, t) Q y(x, t) d x \\
& \quad-(1-d) \int_{l_{1}}^{l_{2}} y^{\mathrm{T}}(x, t-\tau(t)) Q y(x, t-\tau(t)) d x
\end{aligned}
$$

Define $\eta(x, t)=\left[\begin{array}{lll}y^{\mathrm{T}}(x, t) & y_{x}^{\mathrm{T}}(x, t) & y^{\mathrm{T}}(x, t-\tau(t))\end{array}\right.$ $\left.\omega^{\mathrm{T}}(x, t)\right]$; inequality (43) can be also written as follows:

$$
\begin{aligned}
\dot{V}(t) & \leq \int_{l_{1}}^{l_{2}} h_{r i j} \eta(x, t) \\
& \times\left(\begin{array}{cccc}
\Lambda_{r i j} & P B_{r j} K_{D r i} & P A_{d r i} & P C_{r i} \\
* & -(P \Theta+*) & 0 & 0 \\
* & * & -(1-d) Q & 0 \\
* & * & * & 0
\end{array}\right) \eta^{\mathrm{T}}(x,
\end{aligned}
$$


Set $\omega(x, t) \equiv 0$ to obtain the stability analysis results as follows:

$$
\begin{aligned}
\dot{V}(t) & \leq \int_{l_{1}}^{l_{2}} h_{r i j}\left(\begin{array}{c}
y(x, t) \\
y_{x}(x, t) \\
y(x, t-\tau(t))
\end{array}\right)^{\mathrm{T}} \\
& \times\left(\begin{array}{ccc}
\Lambda_{r i j} & P B_{r j} K_{D r i} & P A_{d r i} \\
* & -(P \Theta+*) & 0 \\
* & * & -(1-d) Q
\end{array}\right)\left(\begin{array}{c}
y(x, t) \\
y_{x}(x, t) \\
y(x, t-\tau(t))
\end{array}\right)
\end{aligned}
$$

Based on inequality (37), the fuzzy switched system (36) is stable for any switching signal. The following proof process is similar to Theorem 3; thus it is omitted.

Setting $M_{D r i}=K_{D r i} P^{-1}$ and $N_{r i}=K_{r i} P^{-1}$, following the similar line in Theorem 3, one has

$$
\left.\begin{array}{ccccc}
\Omega_{r i j} & P B_{r j} M_{D r i} P & P A_{d r i} & P C_{r i}-L_{r i}^{\mathrm{T}} S & L_{r i}^{\mathrm{T}} \\
* & -(P \Theta+*) & 0 & 0 & 0 \\
* & * & -(1-d) Q & 0 & 0 \\
* & * & * & -R_{2} & 0 \\
* & * & * & * & R_{1}^{-1}
\end{array}\right)
$$

where

$$
\Omega_{r i j}=\left(P A_{r i}+P B_{r j} N_{r i} P\right)+*+Q
$$

Pre- and postmultiplying both sides of the inequality (46) by matrix

$$
\operatorname{diag}\{X, X, X, I, I\}
$$

where $X=P^{-1}$ and setting $\widetilde{Q}=X Q X$, one can obtain

$$
\left.\begin{array}{ccccc}
\mathrm{H}_{r i j} & B_{r j} M_{D r i} & A_{d r i} X & C_{r i}-X L_{r i}^{\mathrm{T}} S & L_{r i}^{\mathrm{T}} \\
* & -(\Theta X+*) & 0 & 0 & 0 \\
* & * & -(1-d) \widetilde{Q} & 0 & 0 \\
* & * & * & -R_{2} & 0 \\
* & * & * & * & R_{1}^{-1}
\end{array}\right)
$$

where

$$
\mathrm{H}_{r i j}=A_{r i} X+B_{r j} N_{r i}+*+\widetilde{Q}
$$

The matrices $M_{D r i}, N_{r i}, X$, and $\widetilde{\mathrm{Q}}$ can be obtained by solving inequalities (49), then substituting $X, M_{D r i}$, and $N_{r i}$ into

$$
\begin{gathered}
K_{D r i}=M_{D r i} X^{-1}, \\
K_{r i}=N_{r i} X^{-1}
\end{gathered}
$$

we can easily get the controller gains.

\section{Simulation Examples}

Here, we consider the following fuzzy switched parabolic PDE systems with time delay:

$$
\begin{aligned}
& y_{t}(x, t)=\mathscr{A} y(x, t)+\sum_{r=1}^{2} \sum_{i=1}^{2} v_{r}(t) h_{r i}(\theta(x, t)) \\
& \quad \times\left[A_{r i} y(x, t)+A_{d r i} y(x, t-\tau(t))+B_{r i} u(x, t)\right. \\
& \left.\quad+C_{r i} \omega(x, t)\right] \\
& z(x, t)=\sum_{r=1}^{2} \sum_{i=1}^{2} v_{r}(t) h_{r i}(\theta(x, t)) L_{r i} y(x, t)
\end{aligned}
$$

subject to the Dirichlet boundary conditions

$$
\begin{aligned}
& y_{1}\left(l_{1}, t\right)=y_{2}\left(l_{1}, t\right)=0, \\
& y_{1}\left(l_{2}, t\right)=y_{2}\left(l_{2}, t\right)=0,
\end{aligned}
$$

and the initial conditions

$$
\begin{aligned}
& y_{1}(x, 0)=0.5 \sin (2 x), \\
& y_{2}(x, 0)=0.3 \sin (2 x)
\end{aligned}
$$

where

$$
\begin{aligned}
& A_{11}=[3,0 ; 0,3], \\
& A_{12}=[3,0 ; 0,3], \\
& B_{11}=[-1,2 ; 0,-3], \\
& B_{12}=[-1,1 ; 1,-3], \\
& C_{11}=C_{12}=L_{11}=L_{12}=A_{d 11}=A_{d 12}=I,
\end{aligned}
$$

and

$$
\begin{aligned}
& A_{21}=[3,0 ; 0,3], \\
& A_{22}=[-3,0 ; 0,-3], \\
& B_{21}=[1,2 ; 0,-3], \\
& B_{22}=[1,2 ; 1,-3], \\
& C_{21}=C_{22}=L_{21}=L_{22}=A_{d 21}=A_{d 22}=I .
\end{aligned}
$$

The membership functions are chosen as

$$
\begin{aligned}
& h_{11}=h_{21}=\frac{y_{2}(x, t)+1.2}{1.7}, \\
& h_{12}=h_{22}=1-\frac{y_{2}(x, t)+1.2}{1.7}
\end{aligned}
$$

Finally, assume

$$
\begin{aligned}
R_{1} & =[-1,0 ; 0,-1], \\
R_{2} & =[1,0 ; 0,1] \\
S & =[1,0 ; 0,1], \\
\Theta & =[1,0 ; 0,1], \\
\tau(t) & =0.2 .
\end{aligned}
$$




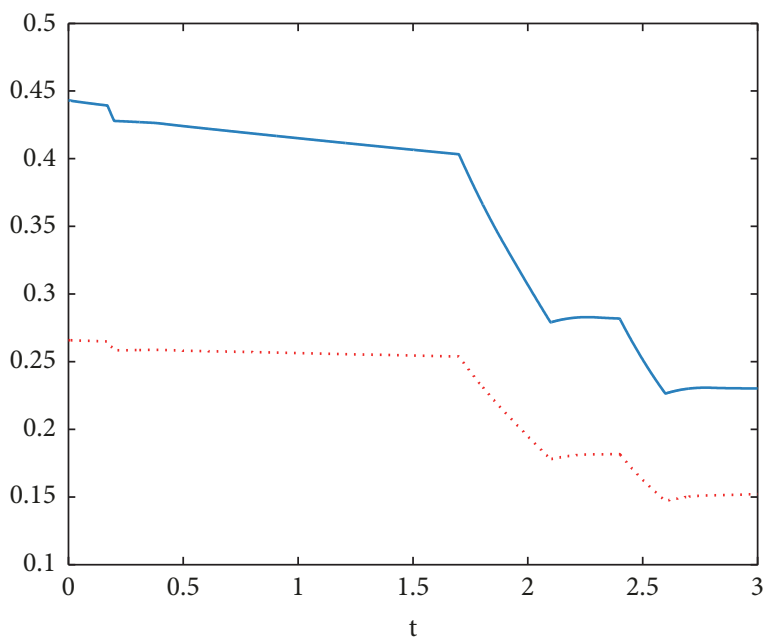

The trajectory of $\left\|\mathrm{y}_{1}(\mathrm{x}, \mathrm{t})\right\|_{2}$ The trajectory of $\left\|\mathrm{y}_{2}(\mathrm{x}, \mathrm{t})\right\|_{2}$

FIGURE 1: The open-loop trajectories of $\left\|y_{1}(x, t)\right\|_{2}$ and $\left\|y_{2}(x, t)\right\|_{2}$.

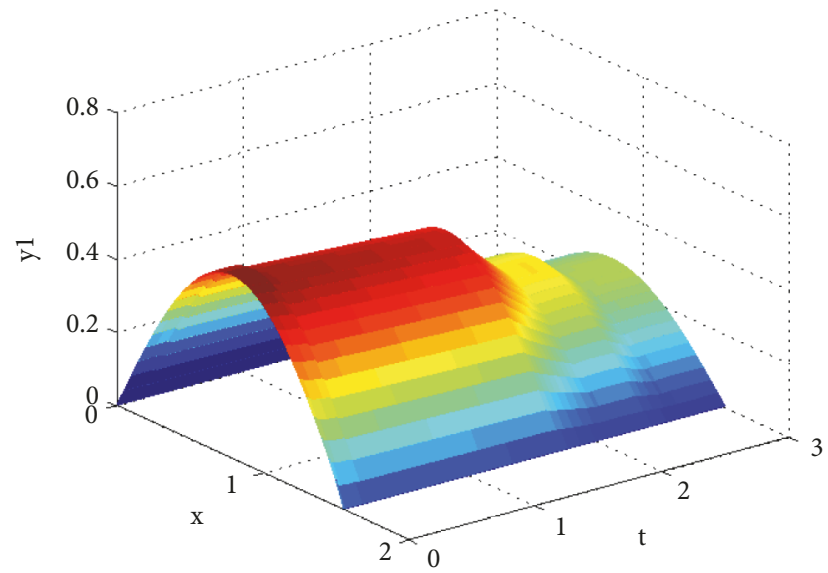

FigURE 2: The open-loop trajectory of $y_{1}(x, t)$.

Now, we give the open-loop trajectories of $\left\|y_{1}(x, t)\right\|_{2}$, $\left\|y_{2}(x, t)\right\|_{2}, y_{1}(x, t), y_{2}(x, t)$, and the switching signal of system (52) in Figures 1-4. It is obvious that the system is unstable, which motives us to design controllers in Examples 7 and 8.

Example 7. In this part, we choose the fuzzy controller as

$$
u(x, t)=\sum_{r=1}^{2} \sum_{i=1}^{2} v_{r}(t) h_{r i}\left(y_{2}(x, t)\right) K_{r i} y(x, t)
$$

By solving the inequality in Theorem 3, we can obtain the controller gains as

$$
\begin{aligned}
& K_{11}=\left[\begin{array}{cc}
3.6601 & 2.8822 \\
-7.8077 & 13.1527
\end{array}\right], \\
& K_{12}=\left[\begin{array}{cc}
4.9581 & -2.8964 \\
-3.6734 & 13.0819
\end{array}\right]
\end{aligned}
$$

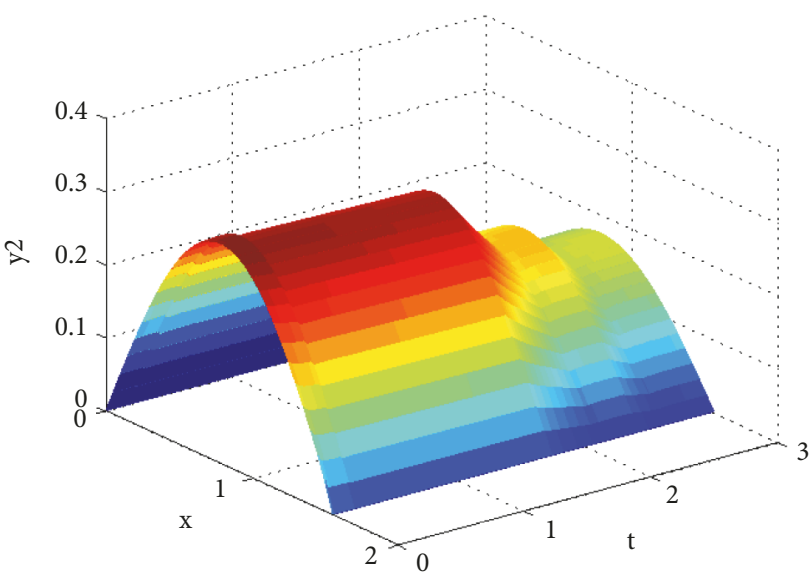

FIGURE 3: The open-loop trajectory of $y_{2}(x, t)$.

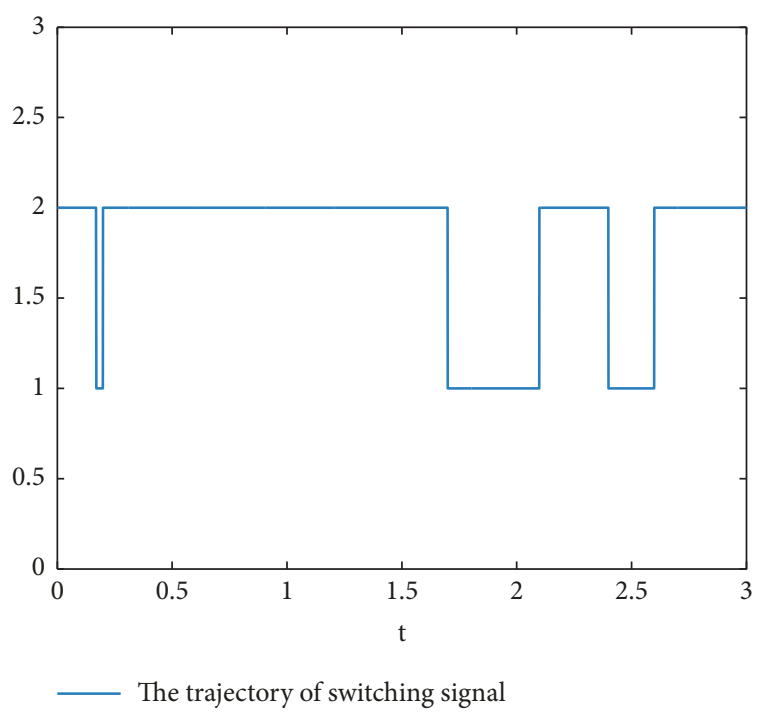

FIGURE 4: The trajectory of switching signal.

and

$$
\begin{aligned}
& K_{21}=\left[\begin{array}{ll}
-5.0199 & 1.9283 \\
-6.5097 & 8.7999
\end{array}\right], \\
& K_{22}=\left[\begin{array}{cc}
-3.0219 & -1.0872 \\
-3.9185 & 4.9103
\end{array}\right]
\end{aligned}
$$

Figures 5-7 present the closed-loop trajectories of $\left\|y_{1}(x, t)\right\|_{2},\left\|y_{2}(x, t)\right\|_{2}, y_{1}(x, t)$, and $y_{2}(x, t)$ of system (52) with the controller (59); the switching signal is shown in Figure 4. It is obvious that the system is stable.

Example 8. In this part, the following fuzzy controller is selected:

$$
\begin{aligned}
& u(x, t) \\
& =\sum_{r=1}^{2} \sum_{i=1}^{2} v_{r}(t) h_{r i}(\theta(x, t))\left[K_{r i} y(x, t)+K_{D r i} y_{x}(x, t)\right]
\end{aligned}
$$




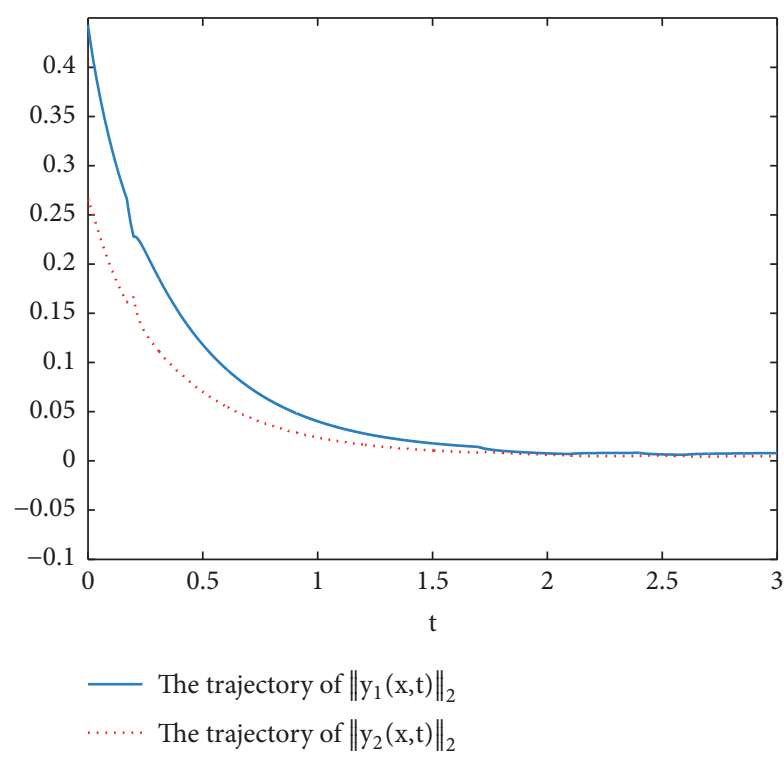

Figure 5: The closed-loop trajectories of $\left\|y_{1}(x, t)\right\|_{2}$ and $\left\|y_{2}(x, t)\right\|_{2}$.

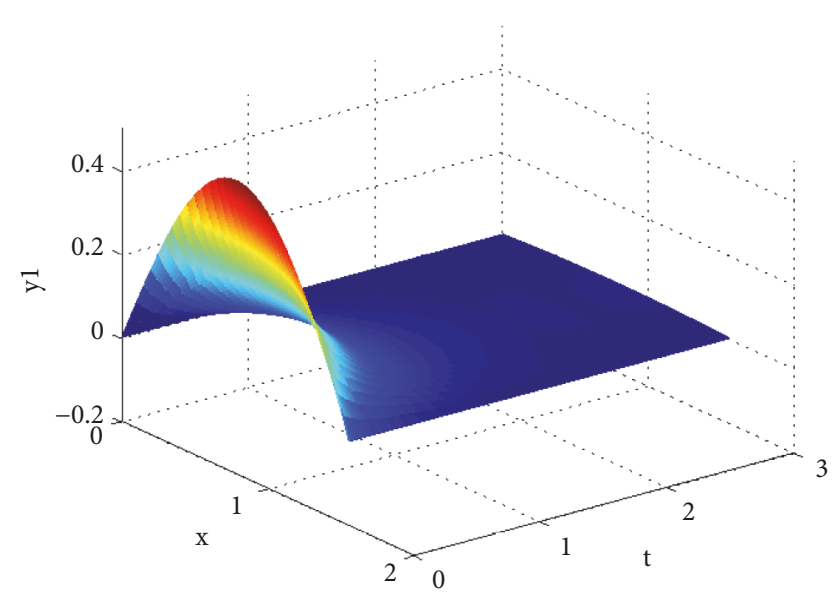

FIGURE 6: The closed-loop trajectory of $y_{1}(x, t)$.

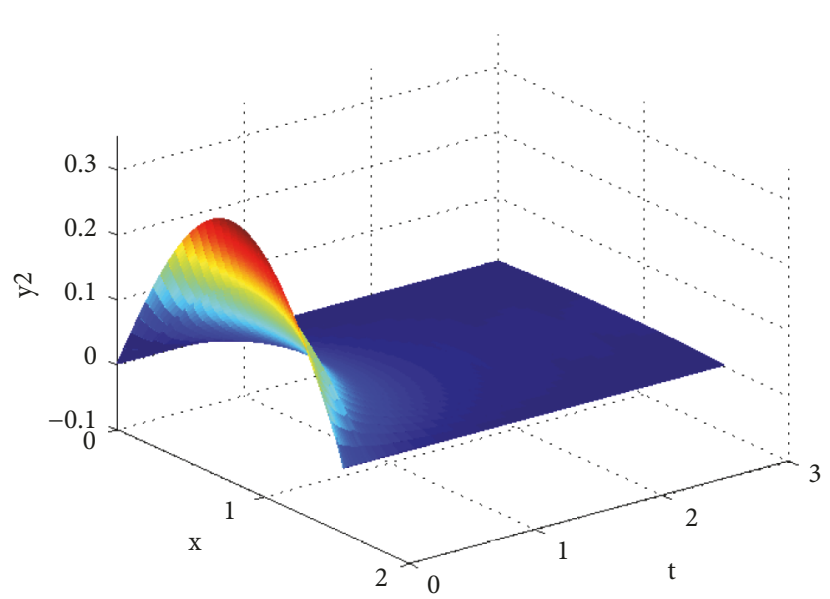

Figure 7: The closed-loop trajectory of $y_{2}(x, t)$.

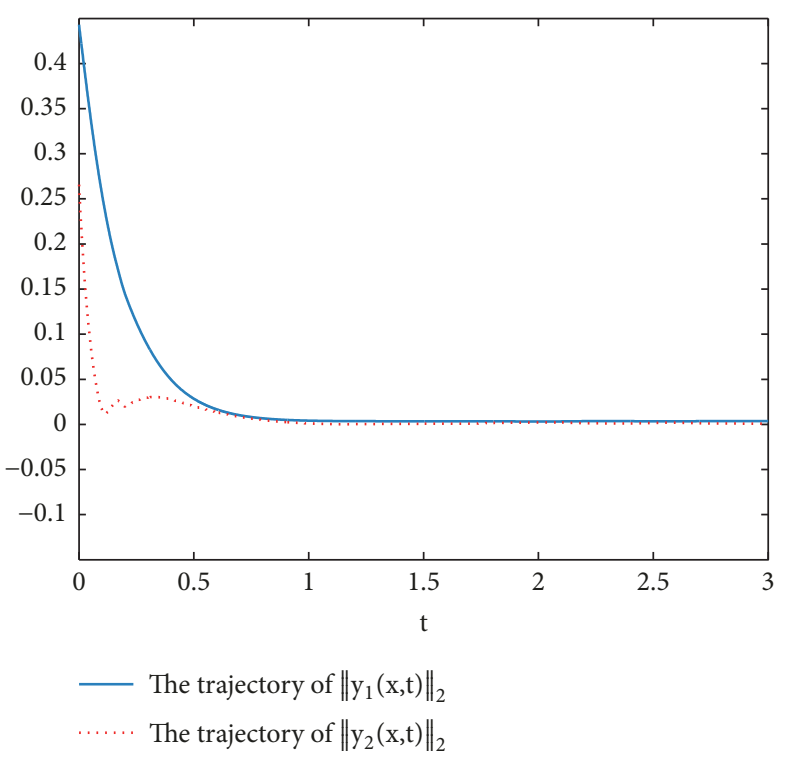

FIgURE 8: The closed-loop trajectories of $\left\|y_{1}(x, t)\right\|_{2}$ and $\left\|y_{2}(x, t)\right\|_{2}$.

Solving inequality in Theorem 6, one can obtain the controller gains:

$$
\begin{aligned}
& K_{11}=\left[\begin{array}{ll}
10.9467 & 3.6839 \\
2.5254 & 3.3732
\end{array}\right], \\
& K_{12}=\left[\begin{array}{ll}
10.9467 & 3.6839 \\
2.5254 & 3.3732
\end{array}\right] \\
& K_{21}=\left[\begin{array}{cc}
-7.1588 & -3.0161 \\
-0.0601 & 2.1910
\end{array}\right], \\
& K_{22}=\left[\begin{array}{cc}
-1.1820 & -0.6319 \\
0.0186 & 0.1704
\end{array}\right]
\end{aligned}
$$

and

$$
\begin{aligned}
& K_{D 11}=\left[\begin{array}{ll}
-1.5514 & -0.4746 \\
-0.3547 & -0.3268
\end{array}\right], \\
& K_{D 12}=\left[\begin{array}{ll}
-1.5514 & -0.4746 \\
-0.3547 & -0.3268
\end{array}\right] \\
& K_{D 21}=\left[\begin{array}{ll}
1.0649 & 0.0780 \\
-0.0160 & 0.2146
\end{array}\right], \\
& K_{D 22}=\left[\begin{array}{ll}
0.9787 & 0.0478 \\
-0.0288 & 0.2191
\end{array}\right]
\end{aligned}
$$

The switching signal is given in Figure 4; Figures 8-10 present the closed-loop trajectories of $\left\|y_{1}(x, t)\right\|_{2},\left\|y_{2}(x, t)\right\|_{2}$, $y_{1}(x, t)$, and $y_{2}(x, t)$, respectively. From Figures 5 and 8 , it is easy to observe that system (52) has better performances via using the fuzzy P-sD controller (62). 


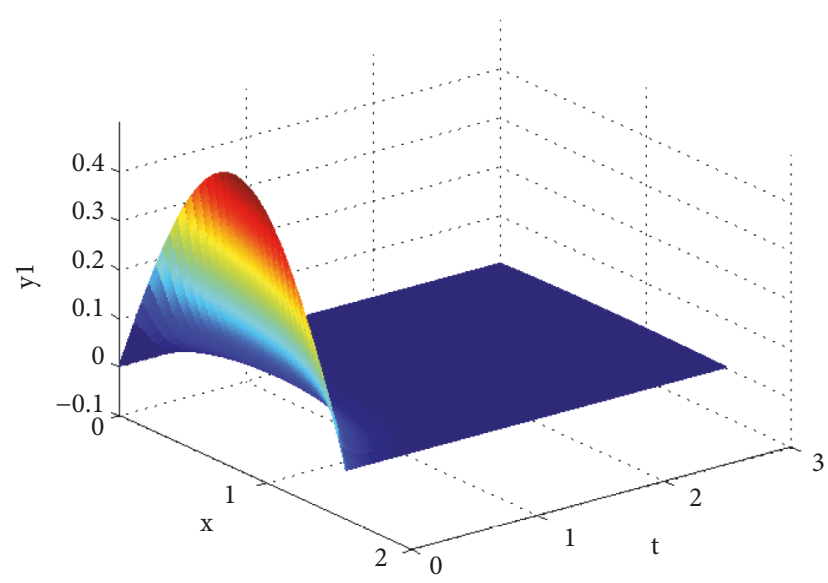

FIGURE 9: The closed-loop trajectory of $y_{1}(x, t)$.

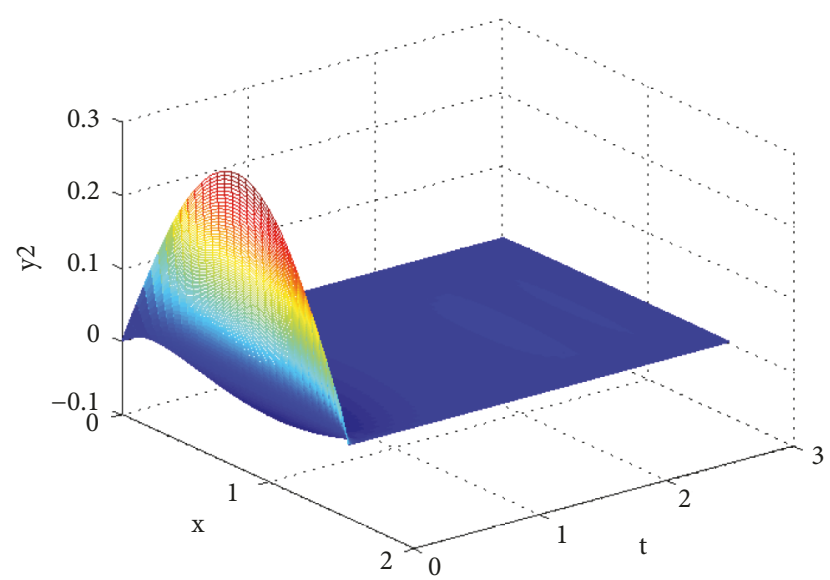

FIGURE 10: The closed-loop trajectory of $y_{2}(x, t)$.

\section{Conclusions}

In this paper, we have developed two fuzzy control laws for a class of T-S fuzzy switched distributed parameter systems (DPSs) with time delay. Using single LyapunovKrasovskii functional, sufficient conditions that ensure the DPSs stable and dissipative were established based on linear matrix inequalities. Furthermore, simulation studies have been shown to support the proposed techniques. It should be stressed that this is only a limited work for fuzzy switched DPSs. In future work, we will further investigate fuzzy control problem for Markovian jump DPSs and multi-Lyapunov functionals will be introduced to reduce the conservatism.

\section{Data Availability}

No data were used to support this study.

\section{Conflicts of Interest}

The authors declare that they have no conflicts of interest.

\section{Acknowledgments}

The project was supported by National Natural Science Foundation of China (nos. U1604146 and 61203047), Science and Technology Research Project in Henan Province (nos. 152102210273 and 162102410024), and Foundation for the University Technological Innovative Talents of Henan Province (no. 18HASTIT019).

\section{References}

[1] W. H. Ray, "Advanced process control," Gerontechnology, vol. 80, no. 9, pp. 1340-1341, 1981.

[2] L. Jadachowski, T. Meurer, and A. Kugi, "Backstepping observers for linear PDEs on higher-dimensional spatial domains," Automatica, vol. 51, pp. 85-97, 2015.

[3] P. D. Christofides, Robust Control of Hyperbolic PDE Systems, Birkhäuser, Boston, Mass, USA, 2001.

[4] A. Smyshlyaev and M. Krstic, Adaptive control of parabolic PDEs, Princeton University Press, Princeton, NJ, USA, 2010.

[5] T. Ahmed-Ali, F. Giri, M. Krstic, L. Burlion, and F. LamnabhiLagarrigue, "Adaptive boundary observer for parabolic PDEs subject to domain and boundary parameter uncertainties," Automatica, vol. 72, pp. 115-122, 2016.

[6] P. Ascencio, A. Astolfi, and T. Parisini, "An adaptive observer for a class of parabolic PDEs based on a convex optimization approach for backstepping PDE design," in Proceedings of the American Control Conference, pp. 3429-3434, 2016.

[7] T. Ahmed-Ali, F. Giri, M. Krstic, F. Lamnabhi-Lagarrigue, and L. Burlion, "Adaptive observer for a class of parabolic PDEs," Institute of Electrical and Electronics Engineers Transactions on Automatic Control, vol. 61, no. 10, pp. 3083-3090, 2016.

[8] A. Schaum, J. A. Moreno, and T. Meurer, "Dissipativity-based observer design for a class of coupled 1-D semi-linear parabolic PDE systems," in Proceedings of the IFAC Workshop on Control of Systems Governed by Partial Differential Equations, pp. 98-103, 2016.

[9] G. Hagen and I. Mezić, "Finite-Dimensional decentralized control of semilinear dissipative parabolic PDEs," Proceedings of the IEEE Conference on Decision and Control, vol. 1, pp. 555560, 2001.

[10] M.-B. Cheng, V. Radisavljevic, and W.-C. Su, "Sliding mode boundary control of a parabolic PDE system with parameter variations and boundary uncertainties," Automatica, vol. 47, no. 2, pp. 381-387, 2011.

[11] X. Zhao, Y. Yin, L. Zhang, and H. Yang, "Control of switched nonlinear systems via T-S fuzzy modeling," IEEE Transactions on Fuzzy Systems, vol. 24, no. 1, pp. 235-241, 2016.

[12] H. D. Choi, C. K. Ahn, P. Shi, L. Wu, and M. T. Lim, "Dynamic Output-Feedback Dissipative Control for T-S Fuzzy Systems With Time-Varying Input Delay and Output Constraints," IEEE Transactions on Fuzzy Systems, vol. 25, no. 3, pp. 511-526, 2017.

[13] H. Li, C. W. Wu, S. Yin, and H. K. Lam, "Observer-based fuzzy control for nonlinear networked systems under unmeasurable premise variables," IEEE Transactions on Fuzzy Systems, vol. 24, no. 5, pp. 1233-1245, 2016.

[14] J. Yu and Z. Yi, "Stability analysis and fuzzy control for uncertain delayed T-S nonlinear systems," International Journal of Fuzzy Systems, vol. 18, no. 6, pp. 1031-1038, 2016.

[15] H. Shen, F. Li, H. Yan, H. R. Karimi, and H. K. Lam, "Finite-time event-triggered H8 control for T-S fuzzy markov jump systems," 
IEEE Transactions on Fuzzy Systems, vol. 26, no. 5, pp. 31223135, 2018.

[16] I. H. Brahim, M. Chaabane, and D. Mehdi, "Fault-tolerant control for T-S fuzzy descriptor systems with sensor faults: an LMI approach," International Journal of Fuzzy Systems, vol. 19, no. 2, pp. 516-527, 2017.

[17] H. Shen, Y. Men, Z. G. Wu, and H. P. Ju, "Nonfragile H8 control for fuzzy markovian jump systems under fast sampling singular perturbation," IEEE Transactions on Systems Man \& Cybernetics Systems, pp. 1-12, 2017.

[18] J. Song, Y. Niu, J. Lam, and H. Lam, "Fuzzy Remote Tracking Control for Randomly Varying Local Nonlinear Models Under Fading and Missing Measurements," IEEE Transactions on Fuzzy Systems, vol. 26, no. 3, pp. 1125-1137, 2018.

[19] X. Song, S. Xu, and H. Shen, "Robust $H_{\infty}$ control for uncertain fuzzy systems with distributed delays via output feedback controllers," Information Sciences, vol. 178, no. 22, pp. 43414356, 2008.

[20] S. Xu and J. Lam, "Robust $H_{\infty}$ control for uncertain discretetime-delay fuzzy systems via output feedback controllers," IEEE Transactions on Fuzzy Systems, vol. 13, no. 1, pp. 82-93, 2005.

[21] H.-N. Wu, J.-W. Wang, and H.-X. Li, "Exponential stabilization for a class of nonlinear parabolic PDE systems via fuzzy control approach," IEEE Transactions on Fuzzy Systems, vol. 20, no. 2, pp. 318-329, 2012.

[22] J. Wang, H. Wu, and H. Li, “Distributed fuzzy control design of nonlinear hyperbolic pde systems with application to nonisothermal plug-flow reactor," IEEE Transactions on Fuzzy Systems, vol. 19, no. 3, pp. 514-526, 2011.

[23] J.-W. Wang and H.-N. Wu, "Guaranteed cost distributed fuzzy control design for a class of nonlinear first-order hyperbolic PDE systems," in Proceedings of the 2012 American Control Conference, ACC 2012, pp. 4375-4380, Canada, June 2012.

[24] H.-N. Wu, H.-Y. Zhu, and J.-W. Wang, " $H_{\infty}$ fuzzy control for a class of nonlinear coupled ODE-PDE systems with input constraint," IEEE Transactions on Fuzzy Systems, vol. 23, no. 3, pp. 593-604, 2015.

[25] H. Yang, B. Jiang, and V. Cocquempot, Stabilization of switched nonlinear systems with unstable modes, vol. 9 of Studies in Systems, Decision and Control, Springer, Cham, 2014.

[26] H. Yang and B. Jiang, "On stability of non-linear and switched parabolic systems," IET Control Theory \& Applications, vol. 7, no. 5, pp. 749-756, 2013.

[27] J. W. Wang, H. N. Wu, and H. X. Li, "Distributed proportionalspatial derivative control of nonlinear parabolic systems via fuzzy PDE modeling approach," IEEE Transactions on Systems, Man, and Cybernetics, Part B, vol. 42, no. 3, pp. 927-938, 2012.

[28] C.-D. Yang, J. Qiu, and J.-W. Wang, "Robust control for a class of nonlinear distributed parameter systems via proportionalspatial derivative control approach," Abstract and Applied Analysis, vol. 2014, Article ID 631071, 8 pages, 2014.

[29] C. Yang, J. Qiu, T. Li et al., "Distributed proportional-spatial derivative control design for 3-dimensional parabolic PDE systems," in Proceedings of the 5th International Conference on Information Science and Technology, ICIST 2015, pp. 583-587, China, April 2015.

[30] T. X. Wang, "Stability in abstract functional-differential equations. I. General theorems," Journal of Mathematical Analysis and Applications, vol. 186, no. 2, pp. 534-558, 1994. 


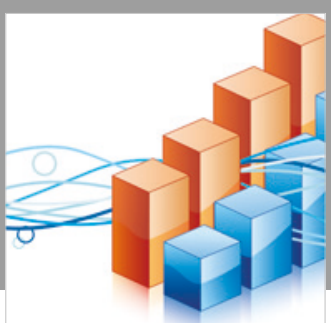

Advances in

Operations Research

\section{-n-m}
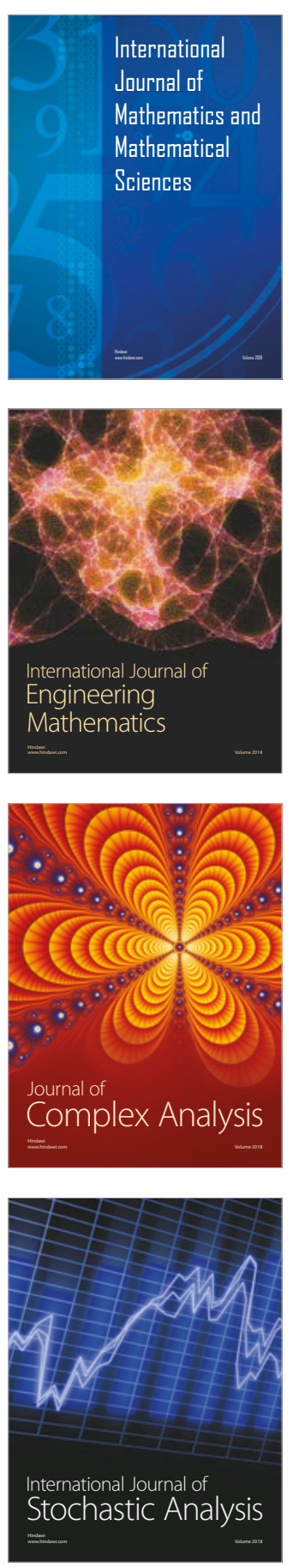
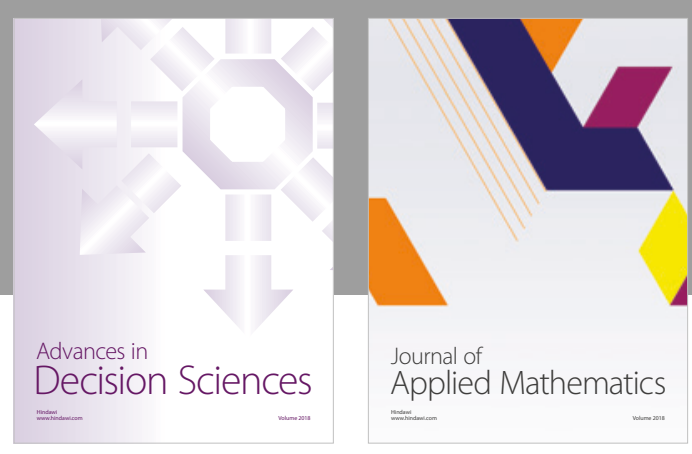

Journal of

Applied Mathematics
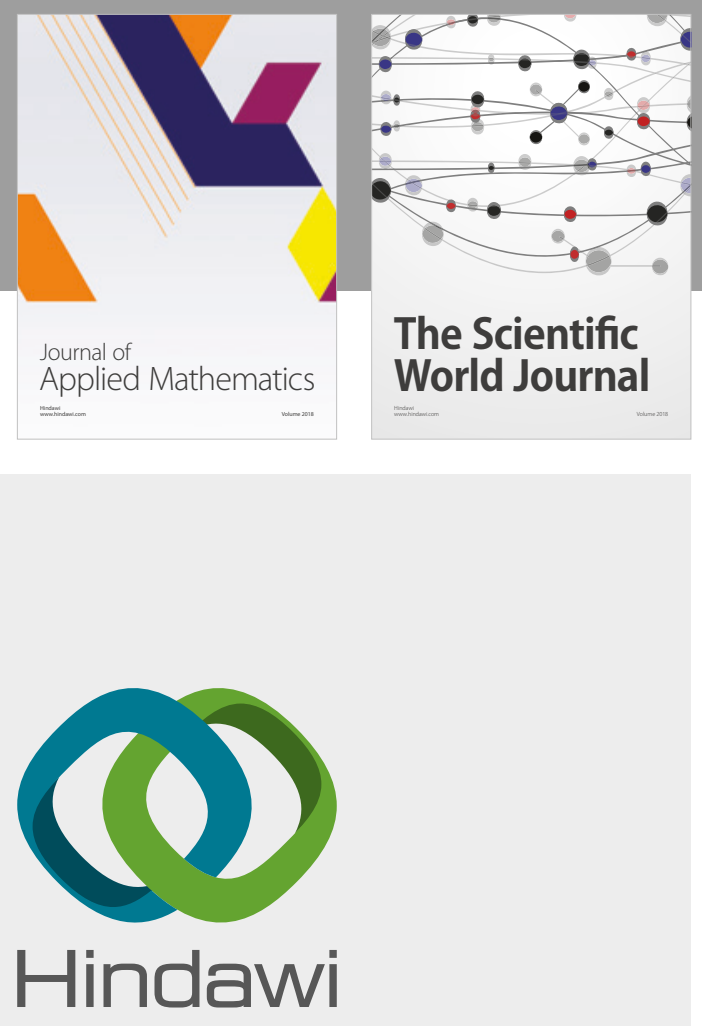

Submit your manuscripts at

www.hindawi.com

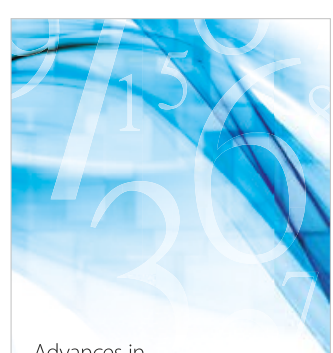

Advances in
Numerical Analysis
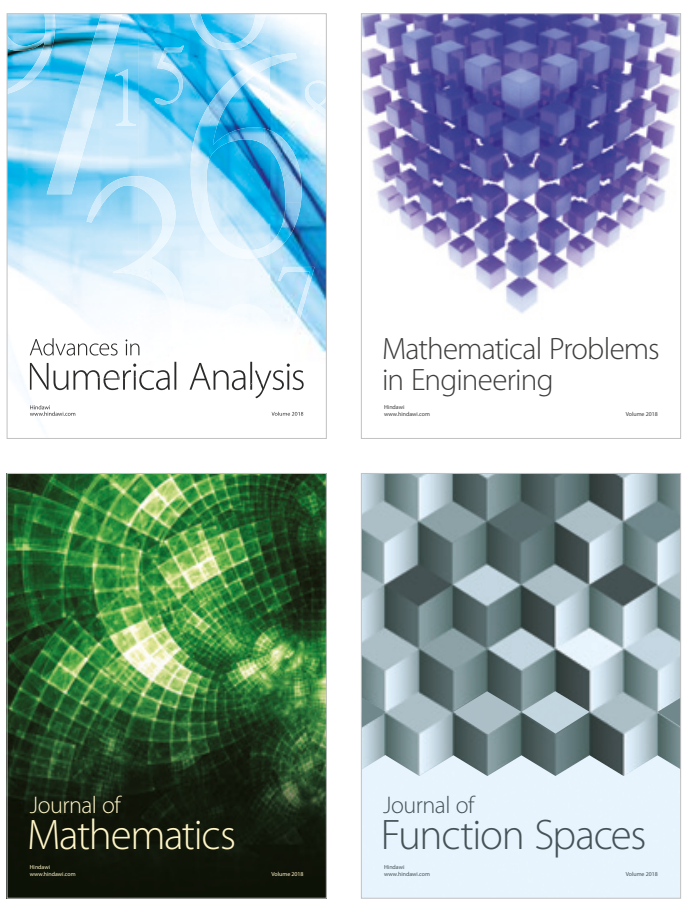

Mathematical Problems in Engineering

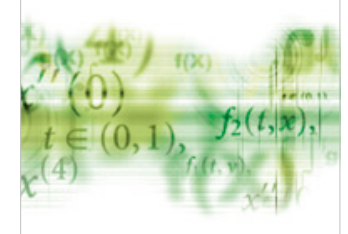

International Journal of

Differential Equations

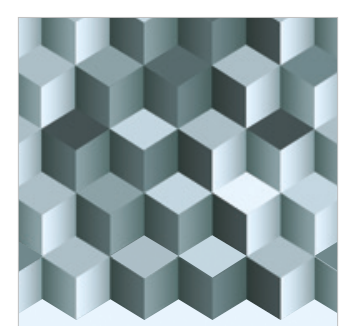

Journal of

Function Spaces
The Scientific

World Journal

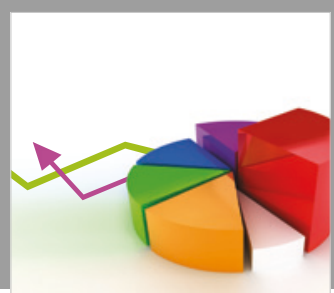

Journal of

Probability and Statistics
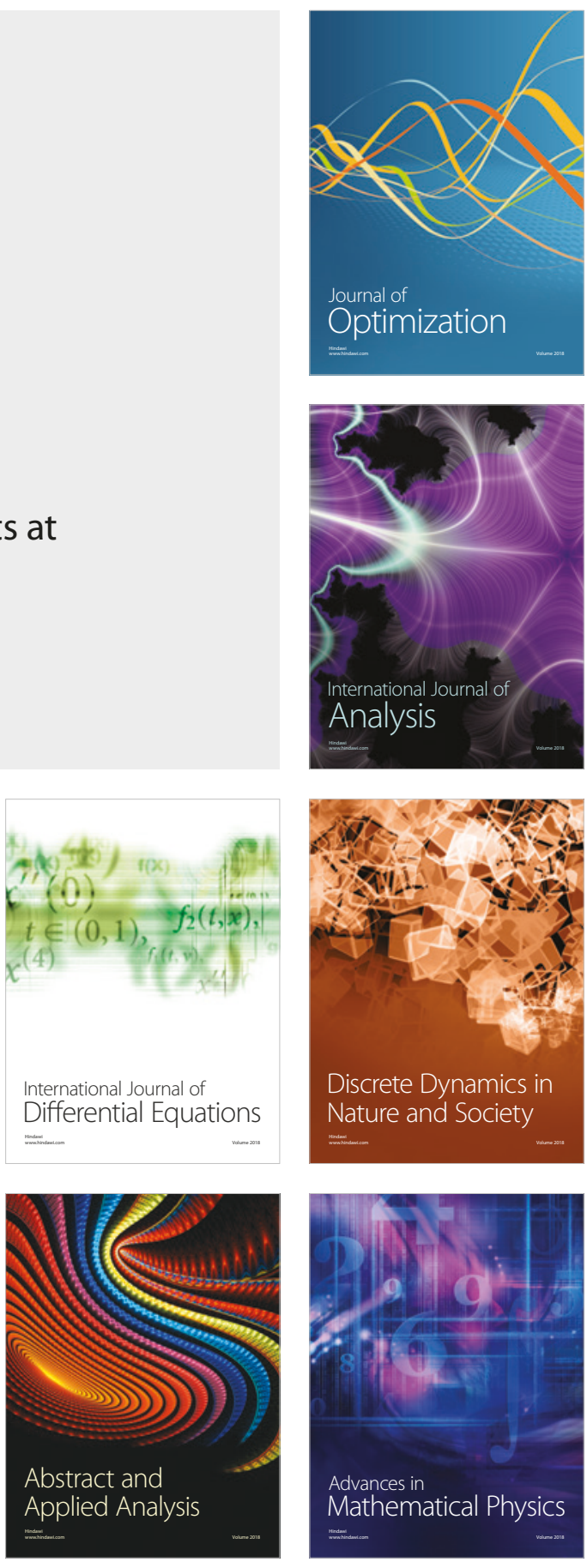